\title{
Confocal laser endomicroscopy is a new imaging modality for recognition of intramucosal bacteria in inflammatory bowel disease in vivo
}

\author{
Driffa Moussata, ${ }^{1}$ Martin Goetz, ${ }^{2}$ Annabel Gloeckner, ${ }^{2}$ Marcus Kerner, ${ }^{2}$ \\ Barry Campbell, ${ }^{3}$ Arthur Hoffman, ${ }^{2}$ Stephan Biesterfeld, ${ }^{4}$ Bernard Flourie, ${ }^{1}$ \\ Jean-Christophe Saurin, ${ }^{1}$ Peter R Galle, ${ }^{2}$ Markus F Neurath, ${ }^{2}$ Alastair J M Watson, ${ }^{3}$ \\ Ralf Kiesslich ${ }^{2}$
}

${ }^{1}$ Department of Gastroenterology, Lyon Sud Hospital, Claude Bernard University Pierre Benite, France ${ }_{2}^{2}$ Medical Clinic, Johannes Gutenberg University of Mainz, Mainz, Germany

${ }^{3}$ Division of Gastroenterology, School of Clinical Sciences, University of Liverpool, Liverpool, UK

${ }^{4}$ Institute of Pathology, Johannes Gutenberg University of Mainz, Mainz, Germany

\section{Correspondence to}

Professor Alastair Watson, Faculty of Health, University of East Anglia, Norwich NR4 7TJ, UK:

alastair.watson@uea.ac.uk

AJMW and RK share last authorship.

Received 10 March 2010 Accepted 12 August 2010 Published Online First 27 October 2010

\section{UNLOCKA}

This paper is freely available online under the BMJ Journals unlocked scheme, see http:// gut.bmj.com/site/about/ unlocked.xhtml

\begin{abstract}
Background and objectives Interaction of bacteria with the immune system within the intestinal mucosa plays a key role in the pathogenesis of inflammatory bowel disease (IBD). The aim of the current study was to develop a fluorescein-aided confocal laser endomicroscopy (CLE) method to visualise intramucosal enteric bacteria in vivo and to determine the involved mucosal area in the colon and ileum in patients with ulcerative colitis (UC) and Crohn's disease (CD).
\end{abstract}

Methods Initially, E coli strains expressing enhanced green fluorescent protein (pEGFP) were

endomicroscopically imaged in mice. In addition, ex vivo and in vivo imaging of fluorescent human enteric bacteria was performed to specify the distinct endomicroscopic appearance of enteral bacteria. Targeted mucosal biopsies towards endomicroscopic identifiable intramucosal bacteria and negative mucosal areas were prospectively obtained during colonoscopy and correlated with bench-top fluorescence microscopy (FISH) to prove the endomicroscopic visualisation of intramucosal bacteria. Finally, a retrospective analysis as well as a prospective study was performed in patients with UC and CD to confirm the presence and distribution of intramucosal bacteria within the gut.

Results Confocal endomicroscopy was able to identify intramucosal pEGFP E coli in mice and strains of enteric microflora in the mucosa of humans. Using FISH as the gold standard, evaluation of 21 patients showed that CLE had a sensitivity of $89 \%$ and specificity of $100 \%$ to identify intramucosal bacteria. In a retrospective study, 113 patients with CD and UC had intramucosal bacteria significantly more often than 50 control patients (66\% vs $60 \%$ vs $14 \%, p<0.001)$. This result was confirmed in a prospective study in which 10 patients with $C D$ and 10 with UC had a significantly wider distribution of involvement with intramucosal bacteria in the colon and terminal ileum compared with 10 controls $(85.2 \%$ vs $75.9 \%$ vs $16.8 \%$, $\mathrm{p}<0.0001$ ).

Conclusions CLE is a new tool that can image intramucosal bacteria in vivo in patients with IBD. Intramucosal bacteria are found more frequently and with a wider distribution in patients with IBD than in patients with a normal intestine.

\section{Significance of this study}

What is already known about this subject?

- Enteric bacteria play an important role in the pathogenesis of inflammatory bowel disease (IBD)

- Intramucosal bacteria have been identified in patients with IBD by a variety of techniques involving mucosal biopsy

- Confocal endomicroscopy can image bacteria on the mucosal surface but has never been reported to identify bacteria with the gut mucosa

\section{What are the new findings?}

- Confocal laser endomicroscopy (CLE) using intravenous fluorescein as a contrast agent can detect bacteria within the gut mucosa

- Endogenous bacteria seen with CLE have the same appearance as exogenously applied fluorescent bacteria

- Using fluorescence in situ hybridisation (FISH) as a gold standard, CLE detects bacteria with a sensitivity of $89 \%$ and a specificity of $100 \%$

- A significantly greater proportion of patients with Crohn's disease and ulcerative colitis have intramucosal bacteria than patients with a healthy intestine

- Intramucosal bacteria also have a more extensive distribution within the intestine of patients with IBD

\section{How might it impact on clinical practice in the foreseeable future?}

- This new endoscopic technique enables the rapid identification of patients with intramucosal bacteria and will be a valuable tool in the elucidation of the pathogenesis of IBD and the development of new clinical algorithms

\section{INTRODUCTION}

The aetiology of inflammatory bowel disease (IBD) is still incompletely understood, but the enteric flora is believed to play an essential role in its pathogenesis. ${ }^{1-6}$ IBD does not develop in genetically engineered murine models of colitis in a germfree environment but is triggered when commensal bacteria are introduced into the intestine. ${ }^{7-9}$ 
Moreover, diversion ileostomy can prevent chronic inflammation in patients with active Crohn's disease (CD) whereas reinfusion of stool into the bypassed segments of the intestine results in a rapid recurrence of the disease. ${ }^{10} 11$ Immunological studies have also stressed that the majority of patients with IBD develop serological and $T$ cell responses to their own enteric flora. ${ }^{12} 13$ Further evidence implicating the importance of enteric flora in the perpetuation of IBD is that treatment with antibiotics and probiotics has been found to be transiently beneficial and can be used for induction and maintenance of remission in IBD. ${ }^{14-16}$

Bacterial products and genes have previously been demonstrated within or attached to the intestinal mucosa of patients with IBD using a variety of techniques including bacterial culture, PCR, electron microscopy or immunochemistry. ${ }^{17-19}$ However, sampling of the intestinal flora with mucosal biopsies disrupts the integrity and structural organisation of the mucosal architecture, preventing determination of the exact location of bacteria within the mucosa. Thus, it has not been possible previously to observe the interaction between bacteria and the mucosal layer in patients with IBD or healthy individuals in vivo.

Recently, a miniaturised confocal microscope integrated into the distal tip of a conventional colonoscope has been developed. ${ }^{20}$ Confocal laser endomicroscopy (CLE) is the only technology that enables microscopic imaging within the mucosal layer of the gut at subcellular resolution. Although previous case studies have shown that CLE can identify bacteria on the mucosal surface during gastroscopy (eg, Helicobacter pylori) ${ }^{21}$ and colonoscopy, ${ }^{22}$ no examples of recognition of bacteria within the wall of the gut have so far been reported.

The aims of the current study were (1) to develop a method to visualise intramucosal bacteria in animals and humans in vivo and (2) using fluorescein-aided CLE, to determine the proportion of patients with ulcerative colitis (UC) and CD with intramucosal bacteria that can be visualised by CLE and to compare this with patients with normal intestines in a retrospective preliminary analysis and a prospective study (figure 1).

\section{MATERIAL AND METHODS}

\section{Imaging of fluorescent bacteria in mice}

The appearance of enteric bacteria was first investigated using C57BL/6 mice and egfp carrying Escherichia coli. C57BL/6 male mice were anaesthetised with ketamine $(75 \mathrm{mg} / \mathrm{kg})$ and medotomidine $(1 \mathrm{mg} / \mathrm{kg})$ intraperitoneally. Surgery was carried out to exteriorise a segment of small intestine $2 \mathrm{~cm}$ in length as previously described. ${ }^{23}$ The epithelial cells were stained with topically applied acriflavine hydrochloride (3.85 mM) (Sigma, Australia) and imaged with a rigid confocal mini-microscope (Optiscan Five 1, Melbourne, Australia) as previously described. ${ }^{24}$ Excitation of laser light was at $488 \mathrm{~nm}$ and emission was greater than $515 \mathrm{~nm}$. Adherent and invasive E coli HM427, transformed with a plasmid carrying the egfp gene (expression of enhanced green fluorescent protein, pEGFP; BD Biosciences Clontech, Mountain View, USA) without alteration of strainspecific characteristics such as the ability to adhere to human intestinal epithelial cells ${ }^{25}$ and to replicate inside murine macrophages, ${ }^{26}$ were placed at a concentration of $4 \times 10^{8}$ on the luminal surface of the exteriorised intestine for $2 \mathrm{~h}$ prior to imaging. Representative images of intramucosal $E$ coli were obtained varying the imaging plane depth of the laser system up to $50 \mu \mathrm{m}$ from the surface of the intestinal mucosa (see figure 2).
Appearance of fluorescent bacteria in murine intestinal epithelium at confocal microscopy

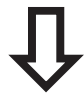

Appearance of patient's faecal bacteria labelled ex vivo and reinstilled into patient $(n=5)$

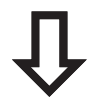

Validation of presence of bacteria at confocal endomicroscopy in human inestine using FISH as gold standard (21 patients, 26 sites)

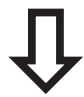

Retrospective preliminary study of frequency of bacteria in patients with UC, Crohn's, and controls

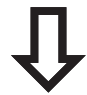

Systematic prospective study of frequency and location of intramucosal bacteria in patients with Crohn's disease, UC and controls.

Figure 1 Study design. FISH, fluorescence in situ hybridisation; UC, ulcerative colitis.

\section{Ex vivo and in vivo imaging of fluorescent human enteric bacteria}

Ten $\mathrm{ml}$ of stool fluids were aspirated in five patients during ongoing colonoscopy. Three $\mathrm{ml}$ of fluorescein 10\% (Novartis Pharma, France) were added and the solution was spun centrifuged $\left(4000 \mathrm{rev} / \mathrm{min}, 10 \mathrm{~min}, 37^{\circ} \mathrm{C}\right.$ ). The supernatant was discarded and $0.1 \mathrm{~g}$ of the pellet was further diluted with $20 \mathrm{ml}$ isotonic saline. This solution was reinjected via the working channel of the confocal colonoscope onto the mucosal surface of the same patient from which the stool was harvested. The whole procedure from aspiration to reinjection took 
Figure $2(A, B)$ Validation of appearances of intramucosal bacteria at confocal endomicroscopy. Fluorescent bacteria on the apical surface of the surface and invading mouse small intestinal epithelium. $E$ coli $(4 \times 108)$ were applied onto the luminal surface for $2 \mathrm{~h}$ prior to imaging with an Optiscan FIVE-1 probe. Epithelial cells are labelled with $3.85 \mathrm{~mm}$ acriflavine. The nuclei are brightly stained (blue arrow). Arrows indicate labelled bacteria on the surface (orange) and within villous epithelial cells (white). Two mice were imaged to obtain representative images of intramucosal bacteria.
A

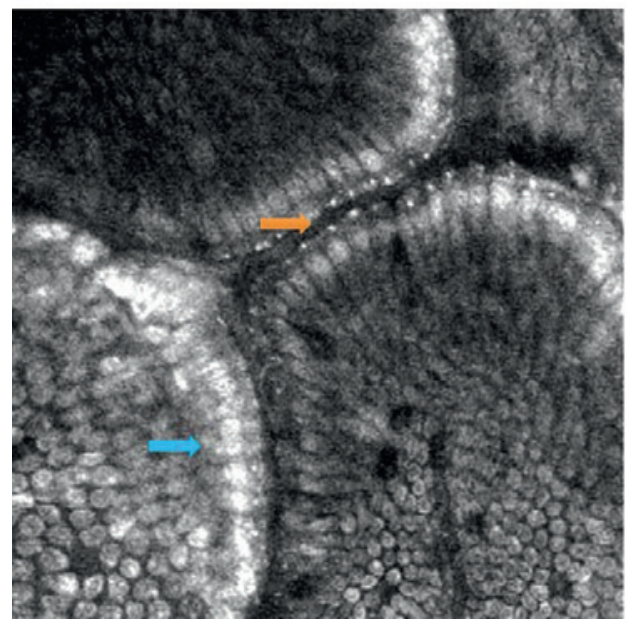

B

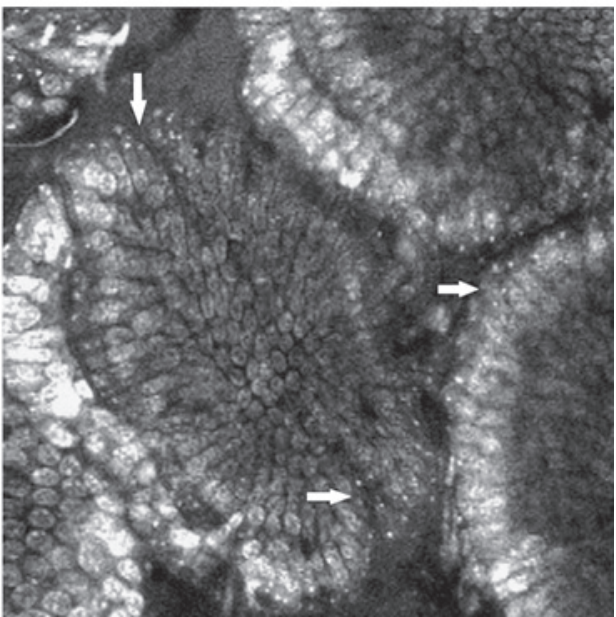

approximately $20 \mathrm{~min}$ and was always performed in a single day. Confocal imaging of the luminal content and the surface of the colonic mucosa was performed to visualise the fluorescent bacteria. Confocal imaging in the lumen, at the mucosal surface and within deeper parts of the mucosa was performed to identify bacterial translocation. ImageJ Version 1.41 (http://www. rsbweb.nih.gov/ij/) was used to measure the diameter of the bacteria.

\section{Fluorescence in situ hybridisation of intramucosal bacteria in humans}

Fluorescence in situ hybridisation (FISH) was performed prospectively on biopsies from different areas (once in the right and left colon) of the colon in 21 patients at 26 sites (4 controls, 13 patients with UC, 3 with CD and 1 with IC; figure 3 ). Sites were selected for targeted biopsies on the basis that endomicroscopic images of the sites either did or did not show bacteria. FISH of bacterial rRNA on glass slides was performed as previously described. ${ }^{27}$ In brief, biopsy specimens were immediately snap frozen in liquid nitrogen and slides with cryosections were fixed in paraformaldehyde and washed in phosphate buffered saline. The universal eubacterial oligonucleotide probe EUB-338 (GCT GCC TCC CGT AGG AGT) and the control probe NONEUB-338 (CGA CGG AGG GCA TCC TCA) complementary to EUB-338 to exclude non-specific binding of the probes were synthesised and 5-prime labelled with Cy3 (Metabion,
Planegg-Martinsried, Germany). Cryosections were incubated with $25 \mathrm{ng}$ of each oligonucleotide in $50 \mu \mathrm{l}$ of hybridisation buffer (containing 20\% formamide) for $90 \mathrm{~min}$ at $46^{\circ} \mathrm{C}$ before washing with the same stringency. Nuclear counterstaining was performed with DAPI $(100 \mu \mathrm{g} / \mathrm{ml}$, Vector Laboratories, Burlingame, California, USA) for $5 \mathrm{~min}$ at $4^{\circ} \mathrm{C}$.

\section{In vivo recognition and characterisation of intramucosal bacteria in humans}

Retrospective study

We analysed retrospectively the confocal database of the Interdisciplinary Endoscopy Unit of the University of Mainz where all patient characteristics, endoscopic images and endomicroscopic images are stored. We identified all patients examined between July 2003 and February 2007 who presented with UC or CD based on serological, clinical, endoscopic and histological criteria. Only patients in clinical remission were selected (patients with CD had a CDAI $<150^{28}$ and patients with UC had a Lichtiger score of $<9),{ }^{29}$ and at least three optical sites within the colon or ileum outside from visible lesions had to be endomicroscopically investigated. Patients with screening colonoscopy who had not suffered from diarrhoea for at least 2 months before colonoscopy served as controls. The presence or absence of bacteria in the colon was assessed in every optical biopsy (set of confocal images per site) by two blinded investigators. Bacteria were identified on the basis of their size and
Figure 3 Intramucosal bacteria in human colon identified at confocal laser endomicroscopy and fluorescence in situ hybridisation (FISH). (A)

Fluorescent intramucosal bacteria within the lamina propria can readily be identified using fluorescein-aided endomicroscopy. Single crypts are shown with their characteristic round appearance (blue arrow). Single bacteria as well as clustered bacteria (orange arrow) can be identified within the lamina propria between two crypts (pericryptal space). (B) FISH testing confirmed the presence of intramucosal bacteria due to the bright red fluorescence. The nuclei and RNA are shown in blue.
A

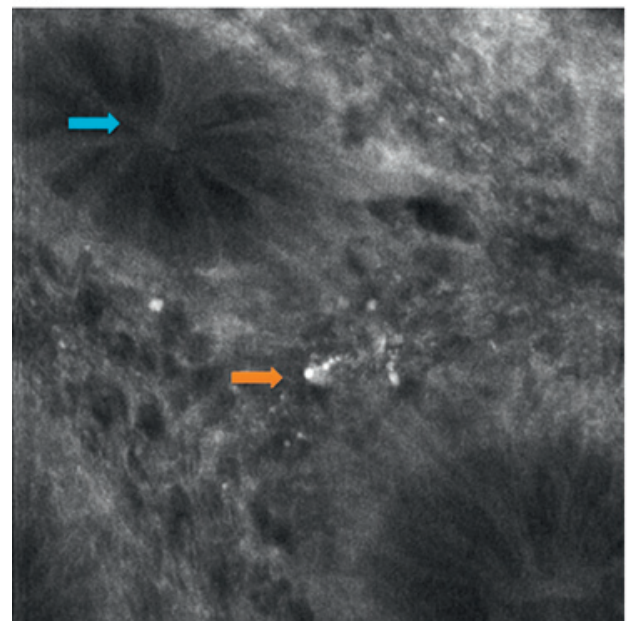

B

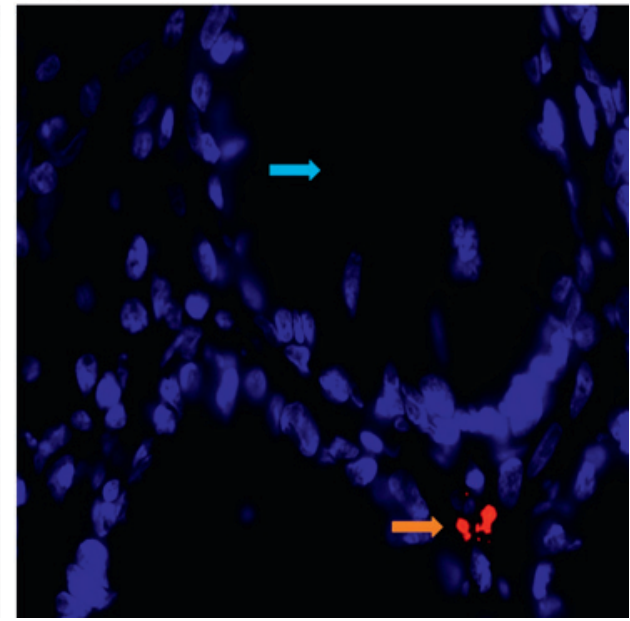


shape (cylindrical, round or spherical; size range $1-2 \mu \mathrm{m}$ ) as specified in previous animal and human experiments. Bacteria were counted within a distinct field of view $(60 \times 60 \mu \mathrm{m})$ which was centred over the highest amount of fluorescence bacterial signal. The amount was judged as follows: no bacteria visible, single bacteria visible, up to 30 bacteria visible, clustered bacteria visible.

\section{Prospective study}

A prospective pilot study was performed on 30 consecutive patients who presented at a single centre (Interdisciplinary Endoscopy Unit of University of Mainz) for colonoscopy because of CD, UC or screening colonoscopy (controls). The study was approved by the local ethical committee. Inclusion criteria were clinically and histologically verified CD or UC in clinical remission. Exclusion criteria were an acute flare with bloody diarrhoea, diarrhoea with $>4$ bowel movements per day, impaired coagulation parameters (Quick $<50 \%$; pTT $>50$ s), impaired renal function (creatinine $>1.2 \mathrm{mg} / \mathrm{dl}$ ), allergy to fluorescein, known gastrointestinal cancer, known stenosis of the ileum or colon, colonic or ileal surgery. Patients were excluded from further analysis if the terminal ileum could not be reached endoscopically or the bowel preparation was insufficient (dropout criteria).

Confocal endomicroscopy was performed as described previously using intravenous fluorescein as a contrast agent. ${ }^{20}$ The colonic mucosa and terminal ileum were examined systematically every $10 \mathrm{~cm}$ and $10-20$ endomicroscopic images from the surface to the deeper parts of the mucosa were obtained from each site.

Intramucosal bacteria were quantified using a method developed as a result of our experience from the retrospective study and FISH testing. Bacteria within the intestinal lumen were not counted. The abundance of intramucosal bacteria was judged based on the affected area within the endomicroscopic field of view $(475 \times 475 \mu \mathrm{m})$. Each image was divided into four quadrants and each quadrant was scored for the presence or absence of bacteria. If no quadrant contained bacteria the image was scored as 'no bacteria', if only one quadrant contained bacteria the image was scored as 'scanty bacteria', and if more than one quadrant contained bacteria it was scored as 'abundant bacteria'.

All images within one optical site were evaluated and the most severe changes were noted. The presence of cellular infiltrates within the lamina propria and crypt distortion based on the Mainz criteria ${ }^{20}$ were also evaluated for each optical site. The conventional endoscopic assessment of macroscopic signs of inflammation was also recorded at each site. Endomicroscopic images were evaluated by an investigator blinded to the history and the endoscopic results of the patients.

\section{Statistical testing}

Absolute and relative frequencies were calculated for qualitative variables in all patient groups; $t$ tests and one-way ANOVA were used to analyse dependencies within two or three patient populations. Regression and multivariate analysis were additionally performed. Statistics were computed using SAS Release 6.08 (SAS Institute Inc).

\section{RESULTS \\ Imaging of fluorescent bacteria in mice}

Our first goal was to determine the appearance at confocal endomicroscopy of bacteria on the surface of the intestinal mucosa and also within the epithelial layer. We first determined the appearance of fluorescent $E$ coli when applied to the mucosal surface of the small intestine of anaesthetised mice. E coli was chosen as an example of intestinal bacteria that are known to invade the intestinal mucosa. We used a strain that has been engineered to express pEGFP. ${ }^{25} 26$ Imaging was undertaken with a rigid confocal mini-microscope (Optiscan Five 1). This instrument has the same confocal scanning technology as the Pentax confocal endomicroscope with which we imaged patients. Thus, the two instruments have identical confocal resolution, depth of plane of focus and image display. Fluorescent $E$ coli could be identified with the mini-microscope in the lumen, on the apical surface of the epithelial cell layer as well as invading into deeper parts of the mucosal layer (figure 2). They had the appearance of intensely fluorescent spots approximately $1-2 \mu \mathrm{m}$ in diameter that could easily be seen against the less intense fluorescence of the acriflavine-stained epithelial and subepithelial cells (figure 2). For comparison, intestinal epithelial cells are approximately $10 \mu \mathrm{m}$ wide.

Bacteria from the human colon were labelled ex vivo with acriflavine and reinstilled into the same patients onto the mucosal surface to establish the typical appearance of bacteria in vivo with confocal endomicroscopy. As shown in figure 4, the bacteria appeared as bright white spots or rods, often in small colonies, similar in appearance to the animal experiments described above.
Figure 4 Appearance of human faecal bacteria labelled ex vivo. Bacteria were harvested from the human colon at colonoscopy, labelled as described in the Materials and Methods section and reinstilled into the human colon. Images were then obtained at confocal laser endomicroscopy. (A) Image of human terminal ileum showing bacteria labelled ex vivo that have entered the epithelial cells (orange arrow). (B) Magnified image.
A

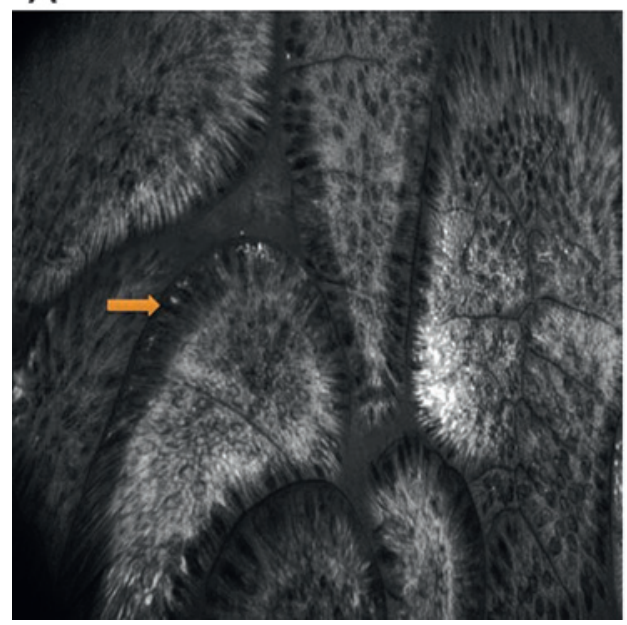

B

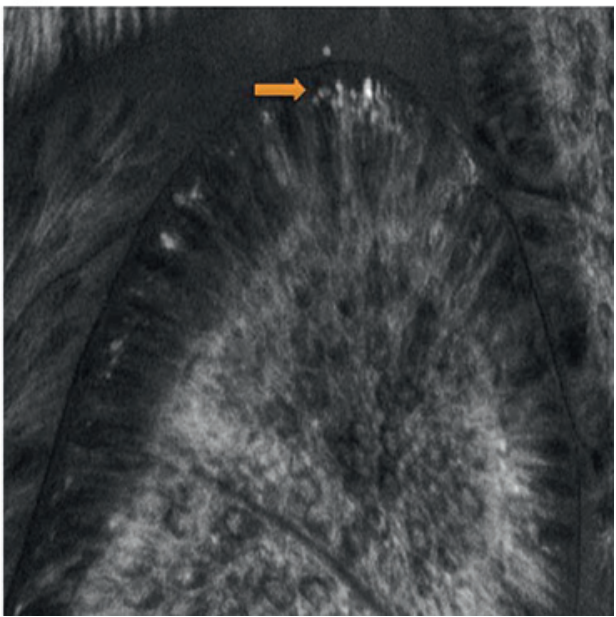




\section{Fluorescence in situ hybridisation of intramucosal bacteria in humans}

To image intestinal bacteria in humans by confocal endomicroscopy, we reasoned that intravenous administration of fluorescein to patients might label intramucosal bacteria since we have previously shown that intravenous fluorescein can leach out of blood vessels and label intestinal epithelial cells. ${ }^{20}$ Using this technique, we found that distinct structures were labelled by fluorescein and appeared within epithelial cells or the lamina propria of the mucosa. These structures strongly resembled intraepithelial and intramucosal bacteria, having the same appearance as pEGFP-expressing bacteria applied to the surface of murine small intestine and human bacteria labelled ex vivo (figures 3 and 4). This suggests that sufficient fluorescein is taken up to label at least a proportion of bacteria in the lamina propria and within epithelial cells.

FISH was used to provide additional validation of the appearance of the bacteria at confocal endomicroscopy, using a universal eubacterial oligonucleotide probe EUB-338 on targeted biopsies of areas we had imaged with confocal endomicroscopy in 21 patients. This probe identifies RNA that is specific to all bacterial species but does not recognise mammalian RNA. Twenty-six sites in 21 patients were selected prospectively for targeted biopsies within $0.5 \mathrm{~mm}$ of the site of endomicroscopic images that either did or did not show bacteria. In 24 out of 26 sites, FISH confirmed the presence or absence of bacteria in all analysed specimens as predicted by endomicroscopy (figure 3$)$. The sensitivity was $89 \%(16 / 18)$ and specificity was $100 \%$ (8/8) for the presence of intramucosal bacteria detected with CLE using FISH as the gold standard. The positive predictive value was $100 \%(16 / 16)$, the negative predictive value was $80 \%(8 / 10)$ and the accuracy was $92.3 \%$ (24/26). Thus, there is a strong correlation between the universal bacterial signal from FISH analysis and the presence or absence of bacteria as identified by CLE. However, it was not possible to identify the bacterial species by either technique.

\section{Frequency of the presence or absence of intramucosal bacteria in humans with and without IBD: retrospective and prospective studies}

We initially undertook a preliminary retrospective study of the frequency of the presence of intramucosal bacteria in 50 control patients, 84 with UC and 29 with CD using images (27062 images analysed) from our database. We found a higher frequency of endomicroscopic identifiable intramucosal bacteria in patients with UC (60\%) and CD (66\%) compared with control patients $(14 \%, \mathrm{p}<0.01)$. In both the CD and UC groups there was no difference in mean Baron score or Rutgeerts' score between patients with or without bacteria (CD: $1.26 \pm 1.15$ vs 1.26 $\pm 1.13, p=0.89$; UC: $2.15 \pm 0.95$ vs $1.80 \pm 1.30, p=0.24)$.

We have previously shown that there is increased capillary leakage of fluorescein at sites of active inflammation. This raises the possibility that the increased numbers of bacteria seen in patients with IBD is an artefact resulting from more fluorescein being available for bacterial uptake, although we mainly examined patients in clinical and endoscopic remission. To evaluate this possibility we determined the average pixel intensity of bacteria and compared this with the average pixel intensity of a $5 \times 5$ pixel region of interest, cells 5 pixels distant from the bacterium. No difference in the pixel intensity of cells or bacteria was found in controls, patients with UC or with CD (figure 5A). We therefore conclude that the increased abundance of bacteria seen in patients with IBD is not an artefact of different concentrations of fluorescein available for bacterial uptake within the tissue.

\section{Prospective study}

Based on our FISH and animal studies, we devised a simple scoring system (visible bacteria per quadrant) for a prospective and systematic study of the frequency of intramucosal bacteria in patients with IBD compared with controls. This scoring system and the systematic endomicroscopic image collection every $10 \mathrm{~cm}$ took account of the substantial heterogeneity of the frequency of intramucosal bacteria observed in our retrospective study.

A total of 6634 images (2622 CD, 2462 UC, 1550 controls) were analysed in 243 optical sites. There were no significant differences between the three different patient groups in age, gender, amount of sedation or average number of images per site. Representative images of intramucosal bacteria from the colon and terminal ileum are shown in figure $5 \mathrm{~B}$. All patients with CD (10/10) and UC $(10 / 10)$ had intramucosal bacteria whereas only $5 / 10$ control patients were positive. We also found a statistically wider distribution of intramucosal bacteria in the colon and terminal ileum in patients with CD or UC with bacteria compared with controls (percentage of positive optical sites $85.2 \%$ vs $75.9 \%$ vs $16.8 \%, p<0.0001$; figure 6 and table 1). A consistent increase in bacteria in patients with IBD compared with controls was found at each $10 \mathrm{~cm}$ segment imaged including the terminal ileum (figure 6). In patients with CD the areas where abundant bacteria were most frequent were the caecum and terminal ileum whereas, in patients with UC, abundant bacteria were frequent in both the rectum and terminal ileum. The distribution of the bacteria in control patients was patchy compared with patients with IBD. There was no correlation between the presence of intramucosal bacteria and macroscopic inflammation visible at endoscopy, crypt distortion and the presence of inflammatory infiltrate. However, there was one significant correlation in patients with CD only; those with CD with leakage of fluorescein into the lumen had significantly more intramucosal bacteria than patients with CD without leakage $(p=0.019)$.

\section{DISCUSSION}

In this study we have developed a new confocal endomicroscopic technique for the identification of patients with colonic or ileal intramucosal bacteria. Using endomicroscopy, we found that intramucosal bacteria are found more frequently in patients with colitis than in patients with a normal colon or terminal ileum. An important feature of confocal endomicroscopy compared with magnifying endoscopy is that, although both technologies can identify surface structures, only confocal endomicroscopy can identify bacteria within the epithelial cells and lamina propria.

Our technique depends on intravenous fluorescein which leaks out of mucosal capillaries and stains intramucosal bacteria which take up fluorescein more avidly than surrounding structures. We have reported previously that there is increased fluorescein leakage in the inflamed intestine. ${ }^{30}$ This raises the possibility that fluorescein might be available within the tissue for uptake by bacteria which might lead to more bacteria becoming visible. We found in practice that this was not the case. As shown in figure $5 \mathrm{~A}$, there was no difference in fluorescent intensity of fluorescein in cells beside bacteria (ie, at a distance of 5 pixels). We found that, when there was substantial fluorescein capillary leakage causing a high fluorescein signal within the epithelial cells, bacteria were not clearly seen. This is because our technique depends on a high contrast in fluorescein fluorescence from the bacteria compared with their cellular environment. Our technique therefore will tend to underestimate the presence 
A

$\mathrm{N}=54$

bacteria

$\mathrm{N}=54$

$\mathrm{N}=52$

From 9

bacteria

bacteria

patients

From 5

From 5

patients

patients

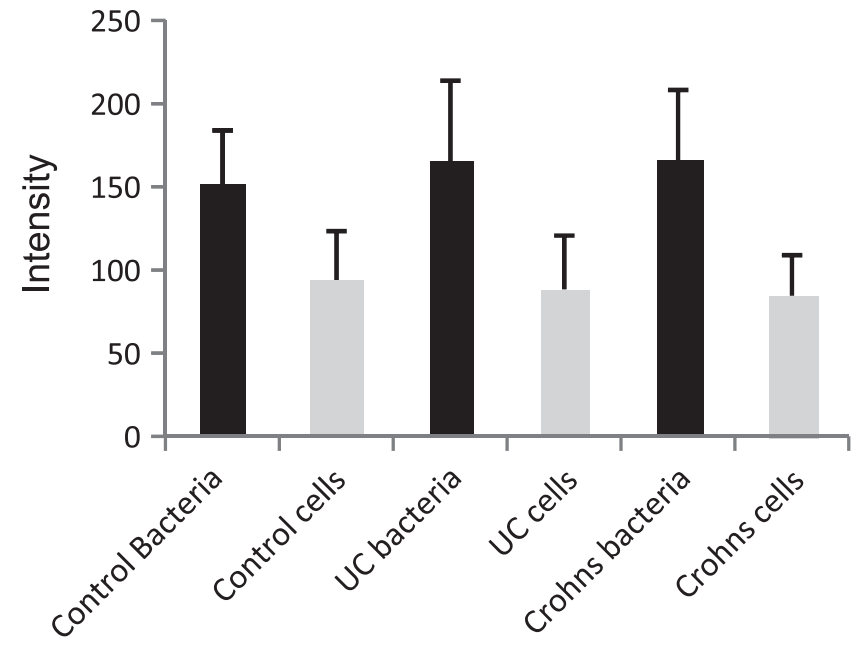

B

Control

Ulcerative Colitis

Crohns
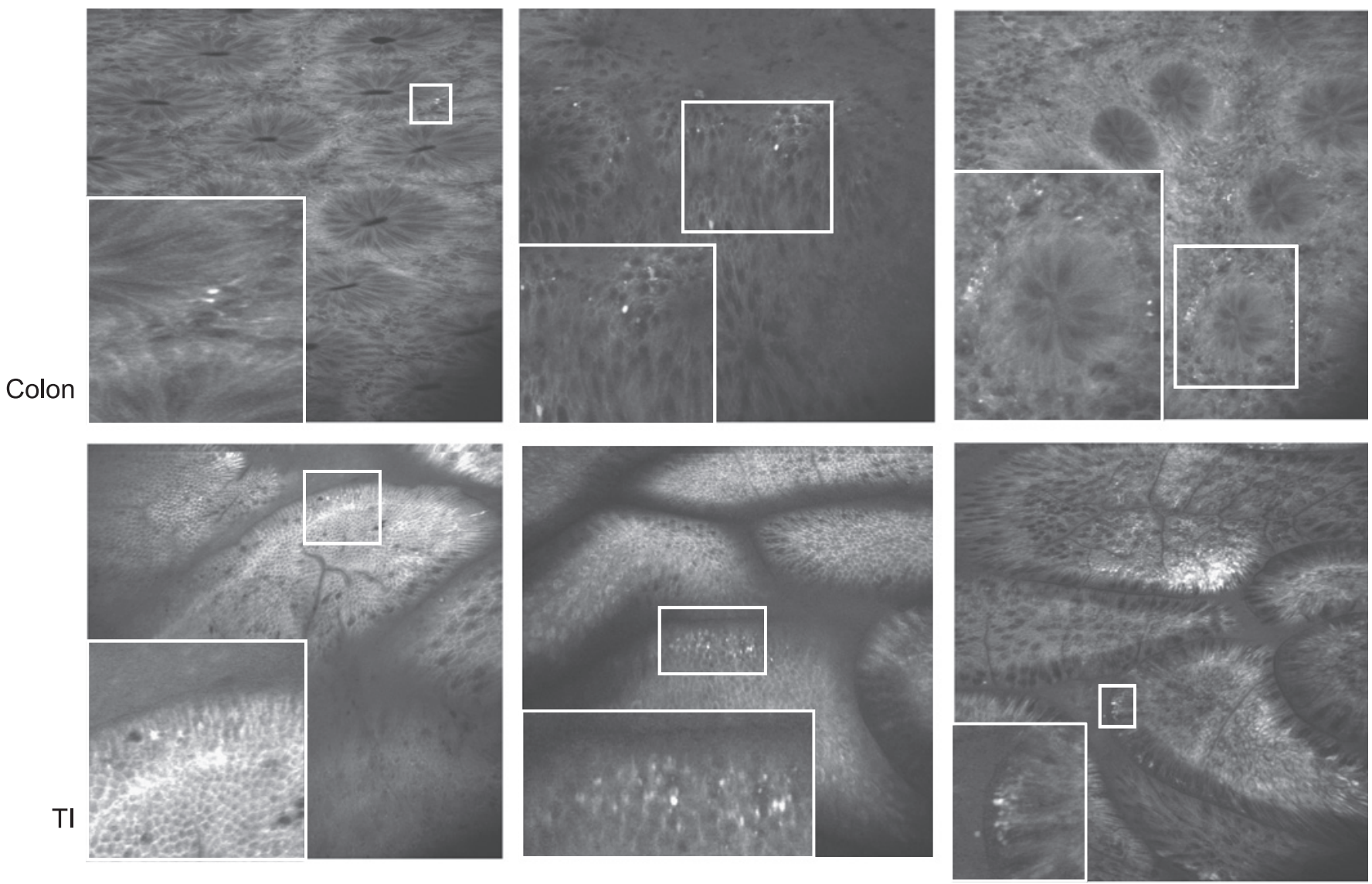

Figure 5 Intramucosal bacteria in control patients, patients with ulcerative colitis (UC) and patients with Crohn's disease. (A) Average pixel intensity of bacteria and a $5 \times 5$ pixel region of interest 5 pixels away from the bacterium. The number of bacteria and patients examined was as follows: control patients: 54 bacteria from 9 patients; UC: 54 bacteria from 5 patients; Crohn's disease: 52 bacteria from 5 patients. As bacteria are less abundant in control patients, images from more patients had to be analysed to accumulate sufficient bacteria. (B) Examples of intramucosal bacteria from the small intestine and colon of controls, patients with UC and patients with Crohn's disease. An enlarged image of the bacteria within the small square is shown at the lower left of each image. TI, terminal ileum.

of bacteria in areas of intense inflammation. It is thus highly unlikely that the increased prevalence of intramucosal bacteria we observed in patients with IBD is an artefact of capillary leakage of fluorescein. It should be noted that intravenous fluorescein differs from acriflavine applied to the surface of the intestine which stains luminal bacteria such as $H$ pylori directly. ${ }^{19}$ 
$\%$ of optical sites with intramucosal bacteria

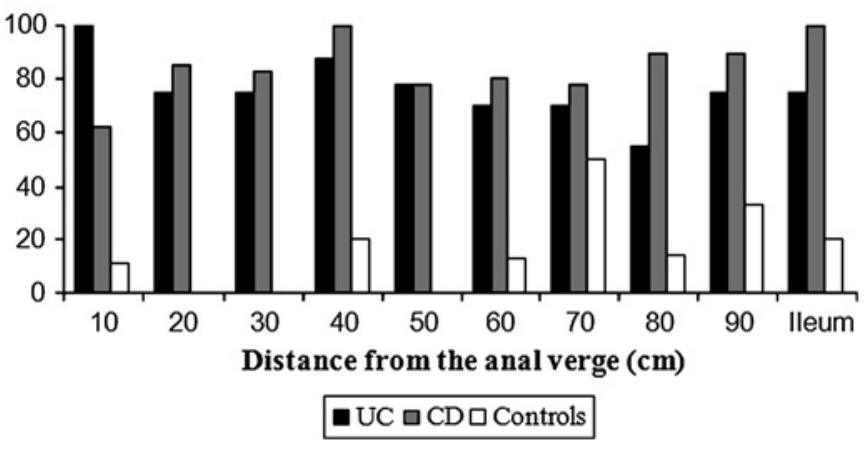

Figure 6 Increased intramucosal bacteria in patients with inflammatory bowel disease. Images were taken systematically at $10 \mathrm{~cm}$ intervals from the anal verge to the terminal ileum and analysed for the presence of bacteria. The percentage of control patients and patients with ulcerative colitis (UC) or Crohn's disease (CD) is plotted at each $10 \mathrm{~cm}$ point. At each segment more patients with UC and CD had intramucosal bacteria than control patients $(p<0.0001$; 10 patients in each group).

Two lines of evidence validate our technique: $E$ coli expressing pEGFP appear as fluorescent spots $1-2 \mu \mathrm{m}$ in diameter both on the apical surface of epithelial cells and within epithelial cells of mouse intestine (figure 2). The rigid confocal endomicroscope (Optiscan) used for these studies contains the same scanning head as the flexible endomicroscope embedded within an endoscope (Pentax) so images from the mouse are the precise equivalent to those from human endomicroscopy. Intramucosal bacteria imaged by confocal endomicroscopy look similar to pEGFP-labelled E coli. Furthermore, faecal bacteria harvested from patients and stained with acriflavine ex vivo also have the same appearance as intramucosal bacteria seen with confocal endomicroscopy (figure 4). Second, we show that CLE identifies intramucosal bacteria with a sensitivity of $89 \%$ and a specificity of $100 \%$ using FISH analysis of biopsies of areas close to $(<5 \mathrm{~mm})$ the confocal images of the mucosa. While there are a variety of artefacts and non-specific appearances on confocal endomicroscopy, there are very few that cause spots $1-2 \mu \mathrm{m}$ in diameter within the epithelial layer. These findings stress the advantage of using CLE compared with other available technologies because bacteria within the lamina propria could only be observed rarely by combining untargeted biopsies and

Table 1 Distribution of endomicroscopically identified intramucosal bacteria

\begin{tabular}{lccc}
\hline & $\begin{array}{l}\text { Crohn's } \\
\text { disease }\end{array}$ & $\begin{array}{l}\text { Ulcerative } \\
\text { colitis }\end{array}$ & $\begin{array}{l}\text { Control } \\
\text { group }\end{array}$ \\
\hline $\mathrm{N}$ & 10 & 10 & 10 \\
Optical sites & 79 & 87 & 77 \\
Presence of bacteria & (\% of sites containing & intramucosal bacteria) & \\
Colon and ileum & 85.2 & 75.9 & 16.8 \\
Presence of bacteria (\%) & per single optical sites & $($ distance from anal verge) \\
$10 \mathrm{~cm}$ & 62.5 & 100 & 11.1 \\
$20 \mathrm{~cm}$ & 85.7 & 75.0 & 0.0 \\
$30 \mathrm{~cm}$ & 83.3 & 75.0 & 0.0 \\
$40 \mathrm{~cm}$ & 100.0 & 87.5 & 20.0 \\
$50 \mathrm{~cm}$ & 77.8 & 77.8 & 0.0 \\
$60 \mathrm{~cm}$ & 80.0 & 70.0 & 12.5 \\
$70 \mathrm{~cm}$ & 77.8 & 70.0 & 50.0 \\
$80 \mathrm{~cm}$ & 90.0 & 55.6 & 14.3 \\
$90 \mathrm{~cm}$ & 90.0 & 75.0 & 33.3 \\
lleum & 100.0 & 75.0 & 20.0 \\
\hline
\end{tabular}

FISH analysis ${ }^{36}$ or comparable other techniques like PCR or culture. ${ }^{17}$ 31-35 Fluorescein-guided endomicroscopy could not only localise intramucosal bacteria but also enabled us to identify single bacteria and bacterial clusters within the distinct endomicroscopic field of view. In addition, imaging with CLE is not prone to artefacts secondary to fixation which might interfere with bacterial visualisation. Since CLE does not disrupt the integrity of the epithelial barrier, bacterial contamination of the specimens (eg, by luminal contents) as occurs when taking a biopsy does not occur.

Our study does not attempt to identify individual species or classes of bacteria as endomicroscopy is only able to image the general shape of the bacteria. Bacteria that are unable to take up fluorescein are not imaged with this technique. ${ }^{33}$ Bacteria in the intestinal lumen can also be imaged, but only if there is a loss of intestinal barrier allowing fluorescein to leak into the lumen. We have shown previously that cell shedding induced by the inflammatory mediator tumour necrosis factor $\alpha$ (TNF $\alpha$ ) can cause a loss of barrier function and is likely to occur in IBD. ${ }^{24}$ Nonetheless, labelling of bacteria within the intestinal mucosa identifies at least the majority of bacteria present as we found that CLE has a sensitivity of $89 \%$ for detecting intramucosal bacteria using FISH as the gold standard.

In our preliminary retrospective study we found that more patients with colitis harbour intramucosal bacteria than patients with a healthy terminal ileum and colon. Furthermore, our systematic prospective study clearly showed that more patients with IBD had intramucosal bacteria in the colon and terminal ileum than controls and that the bacteria were more widely distributed (table 1, figure 6). These results point to defects in the defence mechanisms against bacterial invasion in IBD. We did not find any correlation between the presence of intramucosal bacteria and areas of macroscopic inflammation, corroborating previous studies. ${ }^{17} 3132$ 36-41 This may partly be due to bacteria not being clearly seen in areas of inflammation as discussed above, but may also be because there are intervening pathogenic mechanisms between bacterial invasion and the triggering of an inflammatory response. Our observation that intramucosal bacteria can be found in healthy patients-albeit less frequently-lends support to the idea that the presence of intramucosal bacteria, potentially indicating an intestinal barrier defect, ${ }^{42}{ }^{43}$ is insufficient to trigger an inflammatory response. A further difference between patients with IBD and controls may be that their gut microbiota are more prone to invasion.

Our results are in contrast to the results of studies that use different ex vivo detection technologies. ${ }^{17}$ 31-35 These latter found that the number of bacteria on the mucosal surface increases progressively from self-limiting colitis to UC and from UC to CD, being two powers higher than those in healthy controls. ${ }^{34}$ Also, Bibiloni et al have shown that the number of bacteria from biopsies obtained from patients with UC were twice as high as in samples from patients with CD. ${ }^{31}$ Our technique does not attempt to accurately quantify the bacterial number as it only has the resolution to distinguish absent, scanty or abundant bacteria. The marked inter- and intra-patient heterogeneity in bacterial numbers precludes a more quantitative approach. However, within these constraints, patients with $\mathrm{CD}$ had abundant bacteria more often in the caecum and the terminal ileum whereas, in patients with UC, the rectum was mainly affected. This distribution may reflect the known distribution of inflammation in these diseases.

In conclusion, these animal and human data clearly show that bacteria within the intestinal mucosa can be imaged by CLE. We found that intramucosal bacteria are more common in patients 
with UC and CD compared with a patient cohort undergoing screening colonoscopy with no gastrointestinal symptoms. Endomicroscopy seems to be superior to other available technologies because bacteria can be observed in vivo during ongoing colonoscopy.

Funding Wellcome Trust grant WT087768MA to AJM and a grant from Association François Aupetit (AFA) to DM.

Competing interests RK has an unrestricted grant from Pentax Europe and received instruments for free via Optiscan. All other authors have no competing interests.

Patient consent Obtained.

Ethics approval This study was conducted with the approval of Rheinland-Pfalz, Germany, No. 837.321.07

Provenance and peer review Not commissioned; externally peer reviewed.

\section{REFERENCES}

1. Farrell RJ, LaMont JT. Microbial factors in inflammatory bowel disease. Gastroenterol Clin North Am 2002:31:41-62.

2. Podolsky DK. Inflammatory bowel disease. N Engl J Med 2002;347:417-29.

3. Sartor RB. Pathogenesis and immune mechanisms of chronic inflammatory bowel diseases. Am J Gastroenterol 1997:92:5S-11S.

4. Shanahan F. Host-flora interactions in inflammatory bowel disease. Inflamm Bowel Dis 2004;10(Suppl 1):S16-24.

5. Fiocchi C. Inflammatory bowel disease: etiology and pathogenesis. Gastroenterology 1998:115:182-205

6. Colombel JF, Watson AJ, Neurath MF. The 10 remaining mysteries of inflammatory bowel disease. Gut 2008:57:429-33.

7. Dianda L, Hanby AM, Wright NA, et al. T cell receptor-alpha beta-deficient mice fail to develop colitis in the absence of a microbial environment. Am J Pathol 1997:150:91-7

8. Rath HC, Herfarth HH, Ikeda JS, et al. Normal luminal bacteria, especially Bacteroides species, mediate chronic colitis, gastritis, and arthritis in HLA-B27/ human beta2 microglobulin transgenic rats. J Clin Invest 1996;98:945-53.

9. Sellon RK, Tonkonogy S, Schultz M, et al. Resident enteric bacteria are necessary for development of spontaneous colitis and immune system activation in interleukin10-deficient mice. Infect Immun 1998;66:5224-31.

10. D'Haens GR, Geboes K, Peeters M, et al. Early lesions of recurrent Crohn's disease caused by infusion of intestinal contents in excluded ileum. Gastroenterology 1998:114:262-7.

11. Rutgeerts $\mathbf{P}$, Goboes $\mathrm{K}$, Peeters $\mathbf{M}$, et al. Effect of faecal stream diversion on recurrence of Crohn's disease in the neoterminal ileum. Lancet 1991:338:771-4.

12. Duchmann R, Kaiser I, Hermann E, et al. Tolerance exists towards resident intestinal flora but is broken in active inflammatory bowel disease (IBD). Clin Exp Immunol 1995;102:448-55.

13. Macpherson A, Khoo UY, Forgacs I, et al. Mucosal antibodies in inflammatory bowel disease are directed against intestinal bacteria. Gut 1996:38:365-75.

14. Furrie $\mathbf{E}$, Macfarlane S, Kennedy A, et al. Synbiotic therapy (Bifidobacterium longum/ Synergy 1) initiates resolution of inflammation in patients with active ulcerative colitis: a randomised controlled pilot trial. Gut 2005:54:242-9.

15. Rahimi R, Nikfar S, Rezaie A, et al. A meta-analysis of broad-spectrum antibiotic therapy in patients with active Crohn's disease. Clin Ther 2006:28:1983-8.

16. Isaacs K , Herfarth $\mathrm{H}$. Role of probiotic therapy in IBD. Inflamm Bowel Dis 2008;14:1597-605.

17. Swidsinski A, Loening-Baucke $\mathrm{V}$, Lochs $\mathrm{H}$, et al. Spatial organization of bacterial flora in normal and inflamed intestine: a fluorescence in situ hybridization study in mice. World J Gastroenterol 2005;11:1131-40.

18. Glasser AL, Boudeau J, Barnich $\mathrm{N}$, et al. Adherent invasive Escherichia coli strains from patients with Crohn's disease survive and replicate within macrophages without inducing host cell death. Infect Immun 2001;69:5529-37.

19. Chen W, Li D, Paulus B, et al. Detection of Listeria monocytogenes by polymerase chain reaction in intestinal mucosal biopsies from patients with inflammatory bowel disease and controls. J Gastroenterol Hepatol 2000;15:1145-50.
20. Kiesslich R, Burg J, Vieth $\mathrm{M}$, et al. Confocal laser endoscopy for diagnosing intraepithelial neoplasias and colorectal cancer in vivo. Gastroenterology 2004;127:706-13.

21. Kiesslich R, Goetz M, Burg J, et al. Diagnosing Helicobacter pylori in vivo by confocal laser endoscopy. Gastroenterology 2005;128:2119-23.

22. Gunther $\mathbf{U}$, Epple HJ, Heller $\mathrm{F}$, et al. In vivo diagnosis of intestinal spirochaetosis by confocal endomicroscopy. Gut 2008;57:1331-3.

23. Watson AJ, Chu S, Sieck L, et al. Epithelial barrier function in vivo is sustained despite gaps in epithelial layers. Gastroenterology 2005;129:902-12

24. Kiesslich R, Goetz M, Angus EM, et al. Identification of epithelial gaps in human small and large intestine by confocal endomicroscopy. Gastroenterology 2007:133:1769-78

25. Martin HM, Campbell BJ, Hart CA, et al. Enhanced Escherichia coli adherence and invasion in Crohn's disease and colon cancer. Gastroenterology 2004;127:80-93.

26. Subramanian S, Roberts CL, Hart CA, et al. Replication of colonic Crohn's disease mucosal Escherichia coli isolates within macrophages and their susceptibility to antibiotics. Antimicrob Agents Chemother 2008;52:427-34.

27. Becker C, Wirtz S, Blessing M, et al. Constitutive p40 promoter activation and IL-23 production in the terminal ileum mediated by dendritic cells. J Clin Invest 2003;112:693-706

28. Best WR, Becktel JM, Singleton JW, et al. Development of a Crohn's disease activity index. National Cooperative Crohn's Disease Study. Gastroenterology 1976; 70:439-44.

29. Lichtiger S, Present DH, Kornbluth A, et al. Cyclosporine in severe ulcerative colitis refractory to steroid therapy. N Engl J Med 1994;330:1841-5.

30. Kiesslich R, Goetz M, Lammersdorf K, et al. Chromoscopy-guided endomicroscopy increases the diagnostic yield of intraepithelial neoplasia in ulcerative colitis. Gastroenterology 2007;132:874-82

31. Bibiloni R, Mangold M, Madsen KL, et al. The bacteriology of biopsies differs between newly diagnosed, untreated, Crohn's disease and ulcerative colitis patients. J Med Microbiol 2006;55:1141-9.

32. Kleessen B, Kroesen AJ, Buhr HJ, et al. Mucosal and invading bacteria in patients with inflammatory bowel disease compared with controls. Scand J Gastroenterol 2002;37:1034-41.

33. Schultsz C, Van Den Berg FM, Ten Kate FW, et al. The intestinal mucus layer from patients with inflammatory bowel disease harbors high numbers of bacteria compared with controls. Gastroenterology 1999;117:1089-97.

34. Swidsinski A, Ladhoff A, Pernthaler A, et al. Mucosal flora in inflammatory bowe disease. Gastroenterology 2002;122:44-54.

35. Darfeuille-Michaud A, Neut C, Barnich N, et al. Presence of adherent Escherichia coli strains in ileal mucosa of patients with Crohn's disease. Gastroenterology 1998;115:1405-13

36. Swidsinski A, Weber J, Loening-Baucke V, et al. Spatial organization and composition of the mucosal flora in patients with inflammatory bowel disease. J Clin Microbiol 2005;43:3380-9.

37. Ott SJ, Musfeldt M, Wenderoth DF, et al. Reduction in diversity of the colonic mucosa associated bacterial microflora in patients with active inflammatory bowel disease. Gut 2004;53:685-93.

38. Poxton IR, Brown R, Sawyerr A, et al. Mucosa-associated bacterial flora of the human colon. J Med Microbiol 1997;46:85-91.

39. Prindiville T, Cantrell M, Wilson KH. Ribosomal DNA sequence analysis of mucosa-associated bacteria in Crohn's disease. Inflamm Bowel Dis 2004; 10:824-33

40. Seksik P, Sokol H, Lepage P, et al. Review article: the role of bacteria in onset and perpetuation of inflammatory bowel disease. Aliment Pharmacol Ther 2006;24(Suppl 3):11-18.

41. Strober W, Fuss I, Mannon P. The fundamental basis of inflammatory bowe disease. J Clin Invest 2007:117:514-21.

42. Su L, Shen L, Daniel R, et al. Targeted epithelial tight junction dysfunction causes immune activation and contributes to development of experimental colitis. Gastroenterology 2009;136:551-63.

43. Barrett JC, Lee JC, Lees CW, et al; The UK IBD Genetics Consortium and the Wellcome Trust Case Control Consortium 2. Genome-wide association study of ulcerative colitis identifies three new susceptibility loci, including the HNF4A region. Nat Genet 2009:41:1330-4. 